\title{
Heart rate control using beta-blockers for heart failure with atrial fibrillation: more than enough is too much
}

\author{
Takahiro Okumura $^{1} \cdot$ Yuki Kimura ${ }^{1} \cdot$ Toyoaki Murohara $^{1}$
}

Received: 22 June 2019 / Revised: 3 July 2019 / Accepted: 3 July 2019 / Published online: 30 July 2019

(c) The Japanese Society of Hypertension 2019

As Braunwald indicated at the end of the 20th century [1], heart failure (HF) and atrial fibrillation (AF) have become important worldwide issues that need to be resolved. AF frequently complicates HF, and HF is highly comorbid with AF. AF and HF have been suggested to form an accelerated vicious cycle, and causally, AF induces HF and, conversely, HF induces AF. Pathophysiologically, the hypothesized mechanisms by which AF causes HF, include rapid ventricular response and $\mathrm{R}-\mathrm{R}$ variability, and those by which $\mathrm{HF}$ causes $\mathrm{AF}$, include interstitial fibrosis in the myocardium and altered atrial refraction properties [2]. Therefore, adequate heart rate (HR) control and rhythm management are required in HF patients with $\mathrm{AF}$.

Beta-blockers (BBs) are essential cardioprotective agents for patients with HF, especially in those with cardiac dysfunction with reduced ejection fraction (EF) [3]. There is a consensus that higher dose of BBs can be used to improve the prognosis of patients with HF. The MOCHA investigators reported that $\mathrm{BBs}$ resulted in a dose-dependent improvement of left ventricular function and decreases in mortality and hospitalization rates in HF patients with reduced EF (HFrEF) [4]. Similar results have been shown with lower doses of BBs in studies in the Japanese patients. Thus, major guidelines worldwide recommend the uptitration of BBs as long as these are tolerated in patients with HFrEF, especially in those with a high HR [5].

With regard to HR control, McAlister et al. [6] conducted a meta-analysis of the use of BBs for the treatment of $\mathrm{HF}$ and reported that the magnitude of HR reduction was significantly associated with the survival benefit afforded by BBs to HF patients, but the dose of BBs showed no such association. Furthermore, the SHIFT trial that used

Takahiro Okumura

takaoku@med.nagoya-u.ac.jp

1 Department of Cardiology, Nagoya University Graduate School of Medicine, Nagoya, Japan ivabradine, an HR-reducing agent that does not work via the beta adrenoreceptor, has also shown prognostic improvement in HFrEF patients [7]. This finding indicated that the prognostic improvement may be dependent on how much appropriate HR reduction was achieved and not on how much the BBs could be uptitrated.

Some studies investigated the prognostic difference in HF patients based on heart rhythm. Kotecha et al. [8] reported no benefit of BBs with regard to hospital admissions and mortality in the subgroup of HFrEF patients with AF, unlike in those with sinus rhythm. However, this study was a retrospective meta-analysis using somewhat old trials. Cullington et al. [9] clarified that a slower resting ventricular rate is associated with better survival in HFrEF patients in sinus rhythm but not in AF patients. Furthermore, prognostic improvement due to the administration of BBs has not been established in multicenter clinical trials of $\mathrm{HF}$ patients with preserved EF patients common in elderly, female, or AF patients [5]. These results indicate that the impact of HR control on prognosis potentially differs depending on the left ventricular EF or heart rhythm. In the case of patients in sinus rhythm with AF, we may have to change our strategy for HR control.

The European Society of Cardiology guidelines report that the optimal resting HR in patients with $\mathrm{AF}$ and $\mathrm{HF}$ is unknown but may be between 60 and 100 beats per minute [5]. Hudoyo et al. [10] investigated the association between $\mathrm{HR}$ at discharge and cardiovascular outcomes based on the presence or absence of BBs in HF patients with AF. They clarified that the lowest HR tertile was associated with an increased risk of composite outcomes compared to the middle and highest HR tertiles. However, such an association was not observed in patients who were not taking BBs. In this study, three additional limitations need to be considered in the interpretation of these results. (1) The subjects were patients in whom AF was recognized on electrocardiography performed at admission. As noted in the limitations section, the duration of $\mathrm{AF}$ was unknown. Subjects may have included patients with sinus rhythm at 


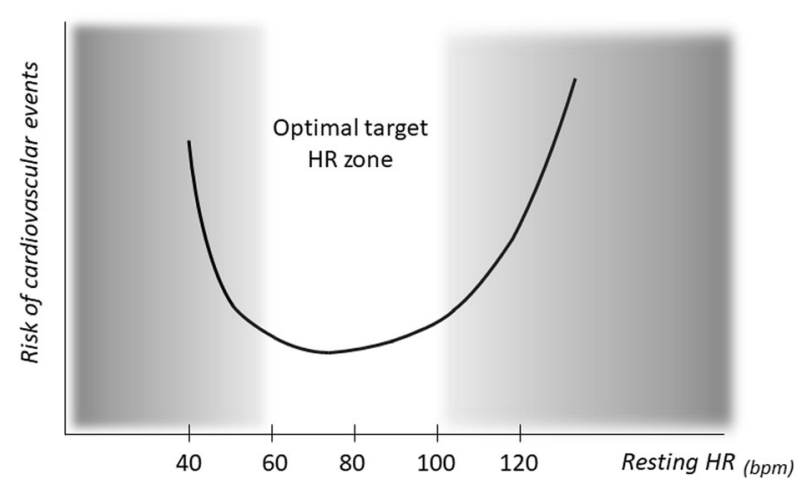

Potential factors associated with optimal target HR determination - Heart rhythm

Heart size (stroke volume)

Systolic function (stroke volume)

Diastolic function / Cardiac relaxation (incomplete relaxation)

Fig. 1 Conceptual schema for determining the optimal target HR in HF patients with AF. The optimal target HR should be set for each patient. When performing HR control using BBs for HF patients with AF, it is necessary to consider heart size, systolic function, cardiac relaxation, and heart rhythm. HR heart rate, HF heart failure, AF atrial fibrillation, BB beta-blocker

discharge. (2) The patients taking BBs had lower fractional shortening than those not taking BBs. That is, the group treated with BBs included many HFrEF patients. However, in the group treated without BBs, fractional shortening was relatively maintained, and the group seemed to contain many HFpEF patients. (3) Some patients with BBs underwent cardiac resynchronization therapy (CRT). The properties of CRT pacing may have affected the rhythms and subsequent prognosis.

When performing HR control using BBs in HF patients with $\mathrm{AF}$, it is necessary to consider heart size, systolic function, cardiac relaxation, and heart rhythm (Fig. 1). For hearts with the same left ventricular EF, the stroke volume is larger when the heart size is larger. The more strongly the ventricular relaxation function is impaired, the higher the need for compensation due to the increase in HR. In such cases, the optimal target HR should be set somewhat higher than usual to preserve cardiac output. Patients taking BBs with HRs $\geq 60$ bpm had slightly fewer cardiac events than those not taking BBs. In patients with HRs $\leq 60 \mathrm{bpm}$, the original effectiveness of the BBs might have been counteracted by the insufficient increase in the stroke volume against bradycardia.

In cases in which the HR is excessively suppressed, an option is to perform device therapy such as CRT, according to the indication criteria to maintain a sufficient HR; however, in the event of the absence of such an indication for device therapy, it may be necessary to reduce the dose of BBs to increase the HR to $\geq 60 \mathrm{bpm}$.

\section{Compliance with ethical standards}

Conflict of interest The authors declare that they have no conflict of interest.

Publisher's note: Springer Nature remains neutral with regard to jurisdictional claims in published maps and institutional affiliations.

\section{References}

1. Braunwald E. Shattuck lecture-cardiovascular medicine at the turn of the millennium: triumphs, concerns, and opportunities. N Engl J Med. 1997;337:1360-9.

2. Schnabel RB, Sullivan LM, Levy D, Pencina MJ, Massaro JM, D'Agostino RB Sr, et al. Development of a risk score for atrial fibrillation (Framingham Heart Study): a community-based cohort study. Lancet. 2009;373:739-45.

3. Yu HT, Yang PS, Lee H, You SC, Kim TH, Uhm JS, et al. Outcomes of rate-control treatment in patients with atrial fibrillation and heart failure - a nationwide cohort study. Circ J. 2018;82:652-8.

4. Bristow MR, Gilbert EM, Abraham WT, Adams KF, Fowler MB, Hershberger RE, et al. Carvedilol produces dose-related improvements in left ventricular function and survival in subjects with chronic heart failure. MOCHA Investigators. Circulation. 1996;94:2807-16.

5. Ponikowski P, Voors AA, Anker SD, Bueno H, Cleland JG, Coats AJ, et al. 2016 ESC Guidelines for the diagnosis and treatment of acute and chronic heart failure: The Task Force for the diagnosis and treatment of acute and chronic heart failure of the European Society of Cardiology (ESC) Developed with the special contribution of the Heart Failure Association (HFA) of the ESC. Eur Heart J. 2016;37:2129-2200.

6. McAlister FA, Wiebe N, Ezekowitz JA, Leung AA, Armstrong PW. Meta-analysis: beta-blocker dose, heart rate reduction, and death in patients with heart failure. Ann Intern Med. 2009;150:784-94.

7. Böhm M, Swedberg K, Komajda M, Borer JS, Ford I, DubostBrama A, et al. Heart rate as a risk factor in chronic heart failure (SHIFT): the association between heart rate and outcomes in a randomised placebo-controlled trial. Lancet. 2010;376:886-94.

8. Kotecha D, Holmes J, Krum H, Altman DG, Manzano L, Cleland JGF, et al. Efficacy of bblockers in patients with heart failure plus atrial fibrillation: an individual-patient data meta-analysis. Lancet. 2014;384:2235-43.

9. Cullington D, Goode KM, Zhang J, Cleland JG, Clark AL. Is heart rate important for patients with heart failure in atrial fibrillation? JACC Heart Fail. 2014;2:213-20.

10. Hudoyo AW, Fukuda H, Imazu M, Shindo K, Fu H, Iwata Y, et al. Heart rate determines the beneficial effects of beta-blockers on cardiovascular outcomes in patients with heart failure and atrial fibrillation. Hypertens Res. 2019. https://doi.org/10.1038/s41440019-0289-4. 